\title{
Scalable flood level trend monitoring with surveillance cameras using a deep convolutional neural network
}

\author{
Matthew Moy de Vitry ${ }^{1,2}$, Simon Kramer ${ }^{2}$, Jan Dirk Wegner ${ }^{3}$, and João P. Leitão ${ }^{1}$ \\ ${ }^{1}$ Department of Urban Water Management, Eawag - Swiss Federal Institute of Aquatic Science and Technology, \\ 8600 Dübendorf, Switzerland \\ ${ }^{2}$ Institute of Environmental Engineering, ETH Zurich, 8093 Zürich, Switzerland \\ ${ }^{3}$ EcoVision Lab, Photogrammetry and Remote Sensing group, ETH Zurich, 8093 Zürich, Switzerland
}

Correspondence: Matthew Moy de Vitry (matthew.moydevitry@eawag.ch)

Received: 14 November 2018 - Discussion started: 15 February 2019

Revised: 3 October 2019 - Accepted: 9 October 2019 - Published: 15 November 2019

\begin{abstract}
In many countries, urban flooding due to local, intense rainfall is expected to become more frequent because of climate change and urbanization. Cities trying to adapt to this growing risk are challenged by a chronic lack of surface flooding data that are needed for flood risk assessment and planning. In this work, we propose a new approach that exploits existing surveillance camera systems to provide qualitative flood level trend information at scale. The approach uses a deep convolutional neural network (DCNN) to detect floodwater in surveillance footage and a novel qualitative flood index (namely, the static observer flooding index - SOFI) as a proxy for water level fluctuations visible from a surveillance camera's viewpoint. To demonstrate the approach, we trained the DCNN on 1218 flooding images collected from the Internet and applied it to six surveillance videos representing different flooding and lighting conditions. The SOFI signal obtained from the videos had a $75 \%$ correlation to the actual water level fluctuation on average. By retraining the DCNN with a few frames from a given video, the correlation is increased to $85 \%$ on average. The results confirm that the approach is versatile, with the potential to be applied to a variety of surveillance camera models and flooding situations without the need for on-site camera calibration. Thanks to this flexibility, this approach could be a cheap and highly scalable alternative to conventional sensing methods.
\end{abstract}

\section{Introduction}

\subsection{The need for urban pluvial flood monitoring data}

Urban pluvial floods are floods caused by intense local rainfall in urban catchments, where drainage systems are usually not designed to cope with storm events of more than a 10-year return period. Although the full impact of such flood events is difficult to gauge because of reporting and knowledge gaps (Paprotny et al., 2018; van Riel, 2011), some studies estimate the societal cost of small but frequent urban pluvial floods to be comparable to the cost of large, infrequent fluvial flooding events (Y. Jiang et al., 2018; ten Veldhuis, 2011). Additionally, it is generally acknowledged that the frequency of urban pluvial floods will increase under the driving forces of climate change and urbanization (Skougaard Kaspersen et al., 2017; Zahnt et al., 2018).

To cope with urban pluvial flood risk, urban drainage managers must understand long-term flooding trends, design appropriate flood mitigation solutions in the medium term, and provide flood alerts in the short term. Numerical flood modeling is a widely used tool for all of these tasks, but a certain amount of data is needed for modeling. Data pertaining to drainage infrastructure, land use, and elevation are required to construct a model, and rainfall data are required to test the model on past rain events. Additionally, flood monitoring data allow for model calibration, which is essential for improving the accuracy of urban drainage models (Tscheikner-Gratl et al., 2016). However, conventional sensors are ill-suited to urban environments, where vehicles can disturb the flow and vandalism is a high risk. Similarly, 
remote sensing is not able to provide data with sufficient spatiotemporal resolution. The lack of monitoring methods and ensuing data scarcity are frequently decried in the urban pluvial flood modeling community (Gaitan et al., 2016; Hunter et al., 2008; El Kadi Abderrezzak et al., 2009; Leandro et al., 2009). In this context, researchers and practitioners have turned to alternative sources of data such as surveillance footage (Liu et al., 2015; Lv et al., 2018), ultrasonic-infrared sensor combinations (Mousa et al., 2016), field surveys (Kim et al., 2014), social media and apps (Wang et al., 2018), and first-hand reports (Kim et al., 2014; Yu et al., 2016).

Although quantitative information (e.g., water level) is commonly sought for, studies show that even qualitative data are useful for calibrating hydraulic and hydrological models. Van Meerveld et al. (2017) calibrated a bucket-type hydrological model with stream level data transformed into sparse, dimensionless class information, such as might be collected by citizen scientists. To accomplish this, the authors used a genetic algorithm (Seibert, 2000) for parameter estimation and the Spearman rank correlation coefficient as an objective function. In doing so, they demonstrated that information could be gained from water level trend data with a conventional calibration toolset. Exploiting a different kind of qualitative information, Wani et al. (2017) showed that binary data from a combined sewer overflow could be used to estimate the parameter value distributions of an urban drainage model. In their research, the Bayesian framework used allowed the reduction of parameter and model uncertainty to be described explicitly. Therefore, we also expect unconventional, qualitative monitoring data to be valuable for the estimation of parameters in urban pluvial flood models.

\subsection{Surveillance cameras as a data source}

Surveillance footage has several advantages when used for flood monitoring. First, many municipalities have already invested in a network of surveillance cameras. In the cities investigated by Goold et al. (2010), these networks usually have fifty to several hundred cameras. In certain cities, however, camera systems operated by institutions are also integrated in the municipal monitoring network. For example, the command and control center for the City of London Police is reported to have access to 60000 cameras (Goold et al., 2010), and the police in Paris have access to 10000 cameras operated by partners (Sperber et al., 2013). The second advantage of surveillance cameras is their high reliability, as their utility for traffic surveillance and crime reduction depends on continuous operation.

However, the use of surveillance footage for flood monitoring has complications. First, camera placement is generally controlled by outside parties for security purposes, thus critical flooding locations may be only partially visible in the footage or even completely missed. Second, the personal privacy of individuals visible in the footage must be protected.
Finally, the interpretation of surveillance footage into a signal that can be assimilated into a flood model is not trivial.

\subsection{Automatic water level monitoring with surveillance images}

While manual reading of water levels from surveillance images is possible (e.g., in the study of Liu et al., 2015), it is both prohibitively labor-intensive at scale and potentially critical from a privacy perspective. Automatic image analysis helps overcome these hurdles, and has already been the subject of research. The following publications provide the current state of the art of automatic water level estimation from ground-level images.

In the work of Lo et al. (2015), video frames are segmented into a number of visually distinct areas using a graphbased approach. The area corresponding to water is identified thanks to an operator-provided "seed", and the water level is qualitatively assessed by comparing the water area to virtual markers placed in the image by the operator. With a more camera-specific solution, Sakaino (2016) estimates water levels with a supervised histogram-based approach which assumes a straight water line on a wall visible in the footage. Similarly, Kim et al. (2011) used a ruler in the camera's field of view as a reference for the water level measurement. A similar approach is used by Bhola et al. (2018), who used the size of large objects like bridges to estimate the real height of automatically detected water surface in the image. Although these methods work well, they rely on in situ measurements and site-specific calibration, and may be challenging to apply to a large number of cameras.

A more modern approach for image-based flood level estimation has been proposed by J. Jiang et al. (2018). The authors use a deep convolutional neural network to extract image features and then apply a regression to infer water level. Although the results are positive, the approach requires that the neural network and regression be retrained for each camera. Thus, the method is probably most valuable for providing redundancy to existing water level readings and not as a scalable flood monitoring solution. Recently, a new approach has been proposed, which theoretically overcomes this problem by estimating water depth from the immersion of ubiquitous reference objects (e.g., bicycles) of known height (Chaudhary et al., 2019; Jiang et al., 2019). However, this approach requires that such objects be visible in the scene in order to provide information.

\subsection{Objective of the present work}

In this work, we propose a novel and highly scalable approach to automatically extract local flood level fluctuations from surveillance footage. By proposing this approach, we aim to provide a tool that can exploit existing surveillance infrastructure to furnish much needed flood information to urban flood modelers and decision-makers. By making scal- 
ability a priority, we hope to facilitate adoption of the tool by practitioners, especially in cities where extensive surveillance camera systems are already in place.

\section{Materials and methods}

Our approach consists in a two-step processing pipeline that combines automatic image analysis with data aggregation (Fig. 1). In a first step, floodwater is segmented in individual video frames with a deep convolutional network (DCNN). The segmented frames are then summarized with an index (namely, SOFI) that qualifies the visible extent and, thereby, the depth of the water over time. We evaluate the performance of this approach using footage from surveillance cameras during various flood events. Additionally, we investigate how the data used to train the DCNN influence both segmentation performance and the information content of the SOFI.

\subsection{Flood water segmentation}

\subsubsection{Image segmentation with deep convolutional neural networks}

Semantic segmentation is the task of annotating each pixel in an image according to a predefined taxonomy. The most recent advances in image segmentation have been made with DCNNs (He et al., 2017), so it is of value to apply this powerful tool to the problem of flood segmentation. DCNNs are a subset of artificial neural networks (ANN), machine learning models with a structure that mimics the structure of neurons in the brain. In the case of DCNNs, images are interpreted through consecutive convolutional (matrix-like) layers that extract and combine information at varying levels of abstraction.

Although the concept of DCNNs originated in the 1980s (Fukushima, 1980), their success for nontrivial problems requires large training sets and computational resources that have only become available relatively recently. An important breakthrough in DCNN development was the fully convoluted network (FCN) introduced by Long et al. (2015), in which the fully connected layers responsible for generating class labels are also formulated as convolutional layers, thereby providing spatially explicit label maps. However, FCN suffered an issue of resolution loss. To solve this issue, Noh et al. (2015) combined FCN with a "deconvolution network", a network that predates FCN (Zeiler et al., 2011) and consists in upsampling and unpooling layers.

\subsubsection{Water segmentation with U-net}

The DCNN architecture used for water segmentation in this work is that of U-net (Ronneberger et al., 2015). U-net builds on the FCN architecture, but differs in that the decoding layers have as many features as their respective encoding layers, which allows the network to propagate context and tex- ture information to the final layers. Additionally, U-net implements "skip" connections to preserve details and object boundaries, by carrying information directly from the encoding to the decoding layers. The U-net architecture is wellsuited to the water segmentation problem because of its relatively compact size compared with more recent state-ofthe-art semantic segmentation architectures, such as Mask R-CNN (He et al., 2017). The smaller size makes it both easier to train with small datasets (like the one available for this study) and faster to run, which is useful for flood monitoring. To code the DCNN, we built on an open-source implementation of U-net (Pröve, 2017) that uses Keras (Chollet et al., 2015) to interface with the TensorFlow library (Abadi et al., 2016).

After exploring a range of hyperparameter values (layer depth, feature size, etc.), we found the following network structure (Fig. 2) to have the best combination of performance and generalization potential for the flood segmentation problem. As input, the network takes color images with a resolution of 512 pixels $\times 512$ pixels. The network is composed of five encoding and five decoding blocks, each block consisting of two convolutions with a kernel size of 3 pixels $\times 3$ pixels. A residual connection around the two convolutions was added to improve the learning capacity of the network (He et al., 2016). A batch normalization layer between each convolution accelerates the training and makes the training performance less dependent on the initial weights (Ioffe and Szegedy, 2015). On the encoding side, the blocks end with a $2 \times 2$ max pooling operation while on the decoding side, blocks start with a 2-D upsampling (or "upconvolution") operation. The skip connections between the encoding and decoding blocks are implemented by taking the final convoluted map of each encoding block and concatenating it to the first map of the corresponding decoding block. The number of features in the first layer is 16 , and the number of features is doubled with increasing layer depth. Additionally, dropout regularization is added between the two deepest convolution layers of the network in order to avoid over-fitting.

\subsubsection{Deep convolutional neural network training strategies}

The collection and labeling of training data is one of the most costly and time-consuming aspects of training DCNNs. For the specific application of flood detection in CCTV and webcam images, training images are particularly rare. Therefore, in this study we evaluated the effectiveness of two strategies for increasing segmentation performance with few training images.

Given the relative rarity of flooding images from surveillance cameras, we used a collection of 1218 labeled images that were collected from the Internet and manually labeled (Chaudhary, 2018). As almost all of the images in the dataset are subject to copyright, we provide a sample of images in the 


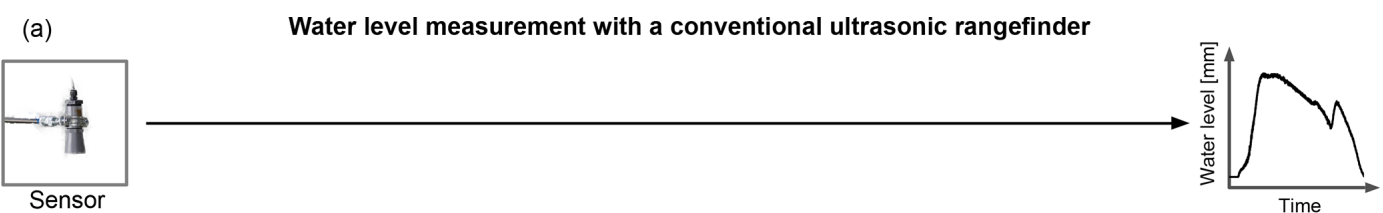

(b)

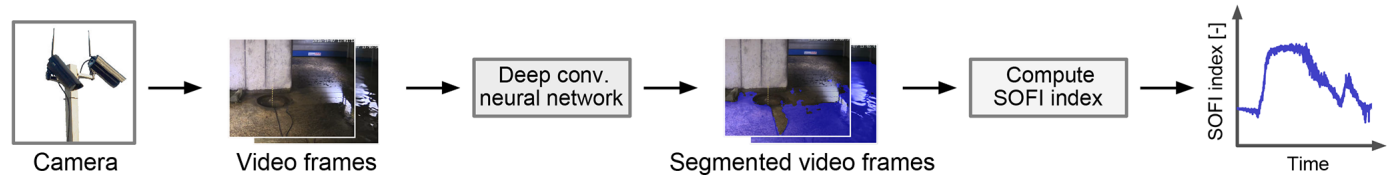

Figure 1. As an alternative to conventional sensors (a), flood trend information is extracted from surveillance footage by computing the fractional water-covered area (SOFI) over a series of video frames (b).

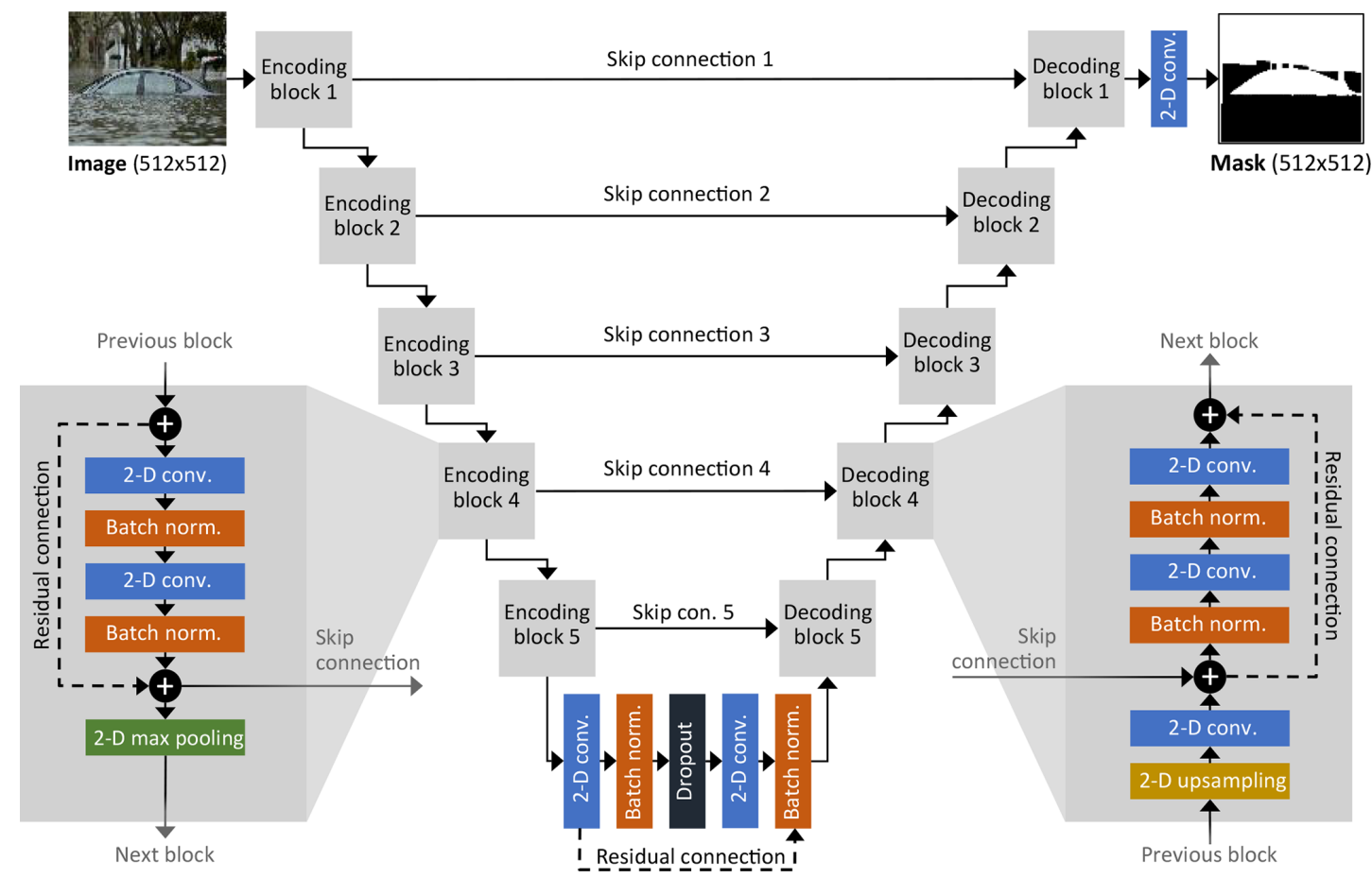

Figure 2. The deep convolutional neural network architecture used to perform water segmentation.

public domain that are representative of the dataset in Fig. 3 . These images have two differences compared with typical surveillance camera images. First, the image quality is generally better in terms of resolution and color reproduction. Second, the pictures almost only depict extensive flooding where most of the ground is covered by water. To provide examples of dry ground, 300 images of street scenery without flooding from the Cityscapes dataset (Cordts et al., 2015) were added to the 1218 Internet images, forming a pool of reference images.

These reference images were used to train a "Basic" version of the DCNN ( $80 \%$ for training, $10 \%$ for validation, and $10 \%$ for testing). Conventional augmentation was applied to the images as they were fed into the network: a random dis- placement of up to $20 \%$ and a random horizontal flip. In this work, we considered two strategies for improving the flood segmentation performance of the Basic training strategy (Table 1).

In the "Augmented" strategy, the same images as for the basic training strategy were used but with additional augmentation steps that degraded image quality to the level of typical surveillance footage. The following image transformations, implemented with the Keras library (Chollet et al., 2015) were applied during augmentation:

- random horizontal mirroring,

- translate image horizontally and vertically by $\pm 20 \%$,

- change in contrast by $\pm 50 \%$, 

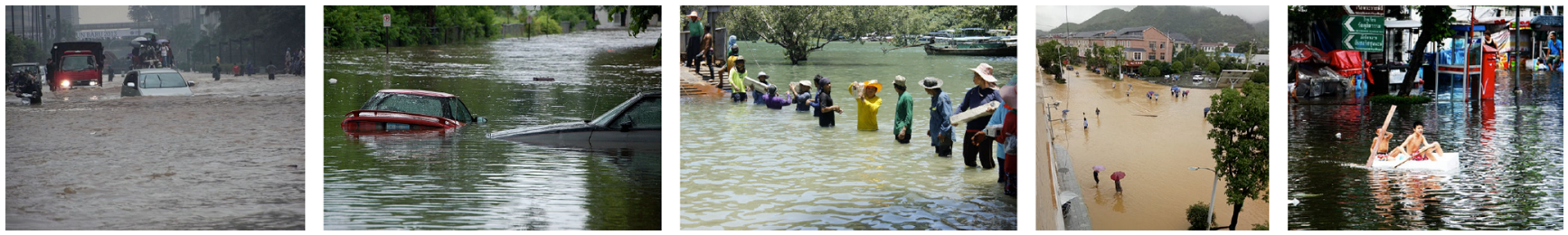

Figure 3. Public-domain images of flooding, representative of the dataset provided by Chaudhary (2018) that was used in this work.

Table 1. Training strategies for the deep convolutional neural network.

\begin{tabular}{|c|c|c|c|}
\hline Strategy & Images used for training & Data augmentation steps & Training steps \\
\hline Basic & $\begin{array}{l}1214 \text { images from Internet } \\
\text { (Chaudhary, 2018) } \\
\text { +300 images from Cityscapes } \\
\text { (Cordts et al., 2015) }\end{array}$ & $\begin{array}{l}\text { Random displacement of up to } \\
20 \% \text {, random horizontal flip }\end{array}$ & $\begin{array}{l}\text { End-to-end training with } 80 \% \\
\text { of images for up to } 200 \text { epochs }\end{array}$ \\
\hline Augmented & Same as Basic & $\begin{array}{l}\text { Same as Basic } \\
+ \text { random Gaussian blur, color } \\
\text { desaturation, contrast modification, } \\
\text { brightness alteration, reduction of } \\
\text { resolution }\end{array}$ & Same as Basic \\
\hline Fine-tuned & $\begin{array}{l}\text { Same as Basic } \\
+ \text { seven frames taken from each } \\
\text { video }\end{array}$ & $\begin{array}{l}\text { No augmentation is applied when } \\
\text { fine-tuning the network. }\end{array}$ & $\begin{array}{l}\text { Retrain Augmented network } \\
\text { with seven manually labeled } \\
\text { video frames }\end{array}$ \\
\hline
\end{tabular}

- resolution deterioration by zooming into the image at different locations up to $33 \%$,

- decreasing saturation by up to $80 \%$,

- alter image brightness between -80 and +20 units,

- blurring with random Gaussian filter.

Augmentation was applied with a $20 \%$ probability each time an image was fed into the network for training (up to 200 times, corresponding to the number of epochs).

In the "Fine-tuned" strategy, we performed transfer learning to adapt the DCNN to specific surveillance videos. This was done by retraining the Augmented network with seven manually labeled frames from each video. ${ }^{1}$ The retraining is performed in two steps. First, only the weights of the last deconvolution block are released and retrained. Then, the rest of the weights are also released and the whole network is retrained. This process resulted in a distinct and specialized network for each footage sequence. In particular, it allowed the networks to learn specific camera characteristics that may not have been represented in the reference images. However, the additional effort required by this training approach limits its practical utility to situations where the increase in data quality is of particularly high value.

To train the network, the adaptive moment estimation (Adam) was chosen as the gradient descent optimizer be-

\footnotetext{
${ }^{1}$ Manual labeling takes around $2 \mathrm{~min}$ per image.
}

cause it shows good convergence properties (Kingma and $\mathrm{Ba}, 2015)$. The dice coefficient served as the loss function, defined after Zou et al. (2004). The DCNN was trained on a Nvidia TITAN X (Pascal) 12 GB GPU. The Basic strategy took approximately $120 \mathrm{~min}$ on average, whereas the Augmented strategy required approximately $180 \mathrm{~min}$ for training. The fine-tuning process required an additional $5 \mathrm{~min}$ of training per video.

\subsection{Static observer flooding index}

The static observer flooding index (SOFI) is introduced in this work as a dimensionless proxy for water level fluctuations that can be extracted from segmented images of stationary surveillance cameras. The SOFI signal is computed as

SOFI $=\frac{\text { \#Pixels }}{\text { Flooded }}$

and corresponds to the visible area of the flooding as seen by a stationary observer. Its value can vary between $0 \%$ (no flooding visible) and $100 \%$ (only flooding visible). When this index is evaluated at multiple consecutive moments in time, the variation of its value provides information about fluctuations of the actual water level under the assumption that the camera remains static and that the view of the flooding is not overly obstructed by moving objects or people. In principle, objects and people will move in and out of the image at a higher frequency than the water level fluctuations, 
so the influence of such obstructions should be limited to an additional noise that can be filtered out. Nevertheless, situations may arise in which the assumption does not hold, for example if an obstruction is permanently removed from the scene during a flood event.

In certain cases, it may make sense to restrict the computation of the SOFI to a region of interest (ROI) of the image. For example, if the image contains more than one hydraulic process, such as accumulation in one part of the image and flow in the other, a ROI can be defined so that the SOFI only reflects the evolution of the accumulation process. The ROI can also be defined to exclude areas in which water segmentation is problematic due to, e.g., unfavorable lighting conditions or visual obstructions. Finally, the ROI can also be chosen over a region of the image in which changes of water level are going to be most visible, e.g., over a vertical wall. In this study, the ROI was implemented by means of a rectangular selection made by the authors according to these criteria. To gauge the effectiveness of this measure, performance was assessed both with and without a user-defined ROI.

\subsection{Performance assessment}

\subsubsection{Surveillance footage}

Six videos depicting flooding were used to assess the performance of the proposed flood monitoring approach. Table 2 provides the characteristics of these videos, which provide a diverse and realistic range of environmental conditions and image qualities.

To assess the performance of the method in terms of flood trend extraction, a reference for the water level trend was established for each video. In the cases of the two FloodX camera videos, a water level signal was available from ultrasonic rangefinders, as documented in (Moy de Vitry et al., 2017). For the other four videos, a qualitative trend was visually estimated with an arbitrary scale. The qualitative trend obtained in this manner was judged sufficient for the present study as this study only investigates the ability of SOFI to predict water level trend, and not the actual water level.

\subsubsection{Flood segmentation performance}

Three images from each video, representing low, medium, and high flooding conditions, were used to assess segmentation performance. Image segmentation is a common classification task that is often evaluated with the mean intersection over union ratio (IoU), also known as the Jaccard index (Levandowsky and Winter, 1971). This metric is applied by running the DCNN on a series of testing images that were not seen during training, and comparing the segmentation result $(S)$ to a manually annotated ground truth $(G)$. The mean IoU is then computed as

$\frac{1}{N} \sum_{i}^{N} \frac{\left|S_{i} \cap G_{i}\right|}{\left|S_{i} \cup G_{i}\right|}$, where $N$ is the number of testing images and $S_{i}$ or $G_{i}$ is the water-covered area in a segmented image or corresponding ground truth image, respectively. The index varies between $0 \%$ for complete misclassification and $100 \%$ for perfect classification.

\subsubsection{Performance of SOFI as a proxy for the water level trend}

To evaluate whether SOFI can be considered a proxy for real water level trends, one can assess the extent to which the relationship between the two signals is monotonic increasing. This quality can be evaluated with the Spearman rank-order correlation coefficient (Spearman, 1904), which is used to measure the degree of association between two synchronous signals. Importantly, it does not assume any other (e.g., linear) relationship between the two signals. To compute the coefficient, the rank of each signal value must be computed relative to its respective signal. For signals in which the same value can appear multiple times (tied ranks), the Spearman rank-order correlation coefficient $\rho$ is given by

$\rho=\frac{\sum\left(x_{i}-\bar{x}\right)\left(y_{i}-\bar{y}\right)}{\sqrt{\sum\left(x_{i}-\bar{x}\right)^{2} \sum\left(y_{i}-\bar{y}\right)^{2}}}$,

where $x_{i}$ and $y_{i}$ are the ranks of the two signals for time step $i$, and $\bar{x}$ and $\bar{y}$ are the average ranks of the SOFI and water level signals, respectively. In the current study, the reference signal for the water level trend was obtained either from an in situ sensor or by visual inspection of the surveillance footage, as described in Sect. 2.3.1. The pandas Python library (McKinney, 2010), which contains an implementation of the Spearman rank correlation coefficient, was used for time series analysis.

\section{Results}

\subsection{Automatic flood water segmentation}

Image segmentation, with the setup described in Sect. 2.1.3, takes around $50 \mathrm{~ms}$ per image. Figure 4 provides sample frames from three of the six surveillance videos used in this work. The other three videos are provided in Fig. S1 of the Supplement. In each case, the human labels as well as automatic segmentations from the various DCNNs are drawn in blue. Additionally, the ROIs are drawn in red for each video, defined manually according to the criteria mentioned in Sect. 2.2.

The basic DCNN was able to detect water in certain cases, but also committed large segmentation errors in the cases of the FloodXCam1, Garage, and Park videos. In the Parking lot video, segmentation appears quite successful, which could be due to the scene being visually similar to the images with which the Basic network was trained. 
Table 2. Surveillance footage used in study.

\begin{tabular}{lllllrlll}
\hline Video & Setting & $\begin{array}{l}\text { Flood } \\
\text { type }\end{array}$ & $\begin{array}{l}\text { Image } \\
\text { resolution }\end{array}$ & $\begin{array}{l}\text { Water } \\
\text { level } \\
\text { trend }\end{array}$ & $\begin{array}{l}\text { Analysis } \\
\text { frequency } \\
\text { (frames min }{ }^{-1} \text { ) }\end{array}$ & $\begin{array}{l}\text { Event } \\
\text { duration }\end{array}$ & Visual clarity of flooding \\
\hline $\begin{array}{l}\text { FloodX } \\
\text { Cam1 }\end{array}$ & $\begin{array}{l}\text { Experimental } \\
\text { facility, weir }\end{array}$ & Artificial & $1280 \times 720$ & Sensor & 60 & 164 min & High: wet surfaces & $\begin{array}{l}\text { Moy de Vitry et al. } \\
(2017)\end{array}$ \\
\hline $\begin{array}{l}\text { FloodX } \\
\text { Cam5 }\end{array}$ & $\begin{array}{l}\text { Experimental } \\
\text { facility, cellar }\end{array}$ & Artificial & $1280 \times 720$ & Sensor & 60 & 166 min & $\begin{array}{l}\text { Medium: wet surfaces, desaturated } \\
\text { image }\end{array}$ & $\begin{array}{l}\text { Moy de Vitry et al. } \\
\text { (2017) }\end{array}$ \\
\hline Garage & Indoor, garage & $\begin{array}{l}\text { Extreme } \\
\text { rainfall }\end{array}$ & $1280 \times 720$ & Visual & 24 & $19.8 \mathrm{~h}$ & $\begin{array}{l}\text { High: moving objects floating in } \\
\text { water }\end{array}$ \\
\hline Park & $\begin{array}{l}\text { Outside, sport } \\
\text { field }\end{array}$ & $\begin{array}{l}\text { River } \\
\text { flooding }\end{array}$ & $640 \times 480$ & Visual & 1 & $13.5 \mathrm{~h}$ & $\begin{array}{l}\text { Low: camera behind window with } \\
\text { reflections; camera is sometimes } \\
\text { jostled; many different lighting condi- } \\
\text { tions throughout the day. }\end{array}$ \\
\hline $\begin{array}{llllll}\text { Cityofchaska (2010) } \\
\text { River }\end{array}$ & $\begin{array}{l}\text { Outside, river } \\
\text { under concrete } \\
\text { bridge }\end{array}$ & $\begin{array}{l}\text { Extreme } \\
\text { rainfall }\end{array}$ & $1280 \times 720$ & Visual & 30 & $6 \mathrm{~h}$ & $\begin{array}{l}\text { Low: camera sometimes submerged; } \\
\text { lens blurry due to water drops; high } \\
\text { image compression }\end{array}$ \\
\hline $\begin{array}{l}\text { Parking } \\
\text { lot }\end{array}$ & Street scenery & $\begin{array}{l}\text { Extreme } \\
\text { rainfall }\end{array}$ & $1280 \times 720$ & Visual & 12 & 200 min & $\begin{array}{l}\text { Low: partially nighttime images; lens } \\
\text { blurry due to water drops }\end{array}$ \\
\hline
\end{tabular}
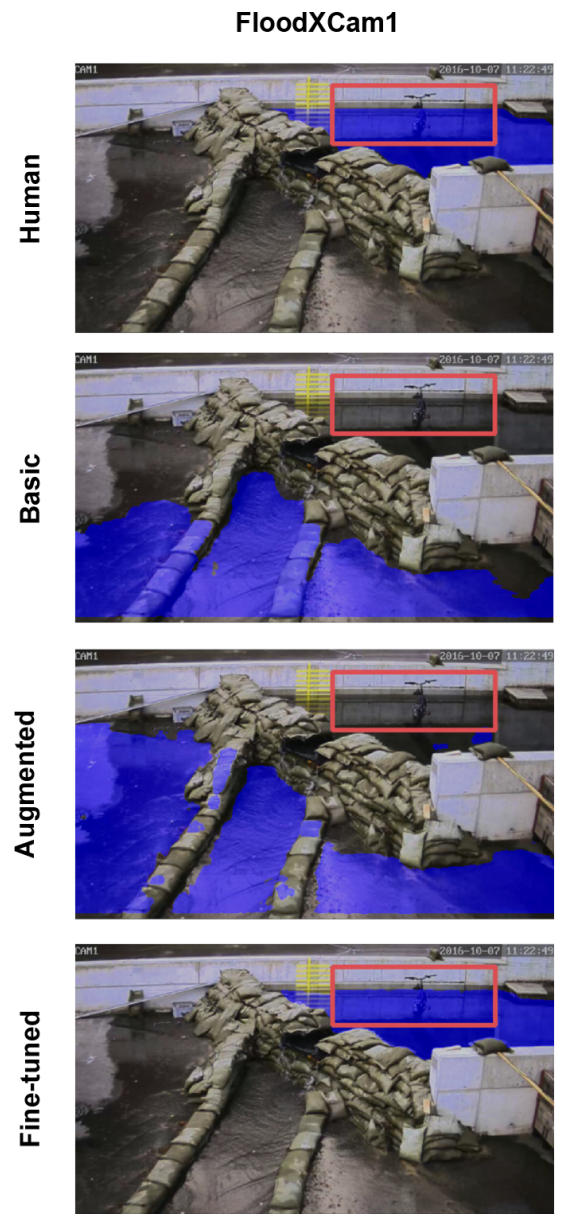

Garage
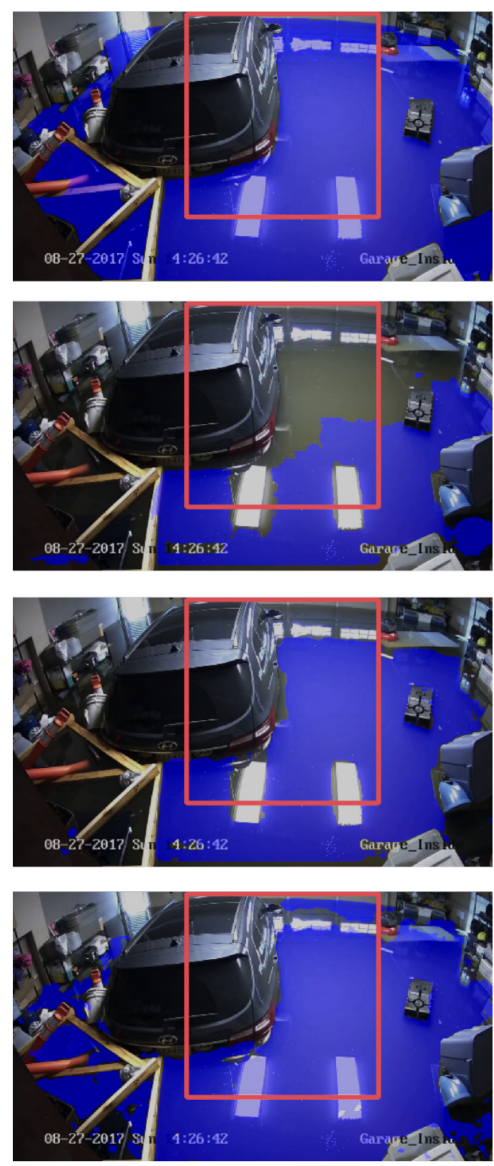

Park
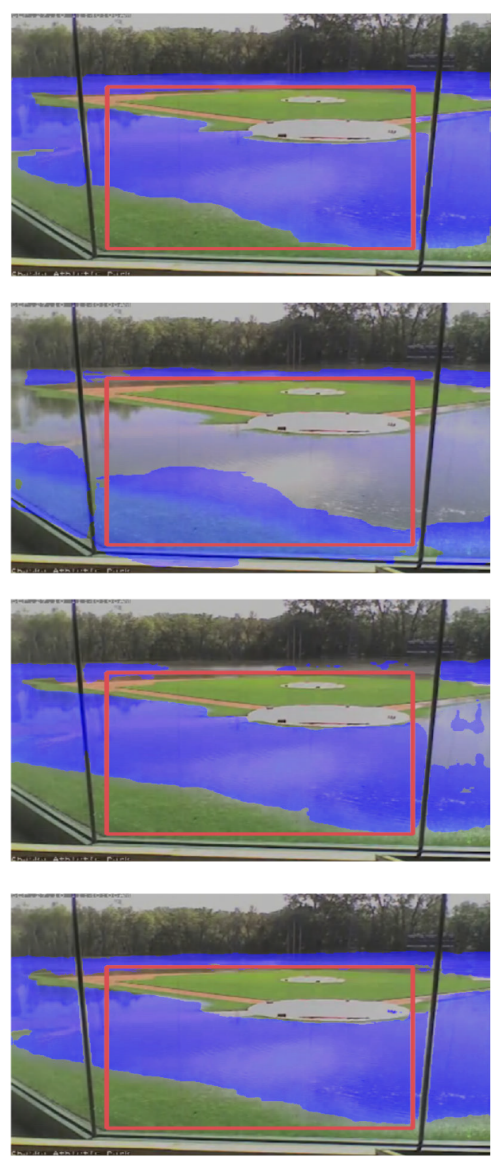

Figure 4. Sample frames taken from three of the six analyzed surveillance videos, shown with the human label or automatic flood segmentation in blue and the regions of interest (ROI) in red. The samples show how the Augmented strategy improves segmentation in the Garage and Park samples, but the segmentation for FloodXCam1 is only successful with the Fine-tuned training strategy. The sample frames from the remaining three videos are provided in the Supplement. 


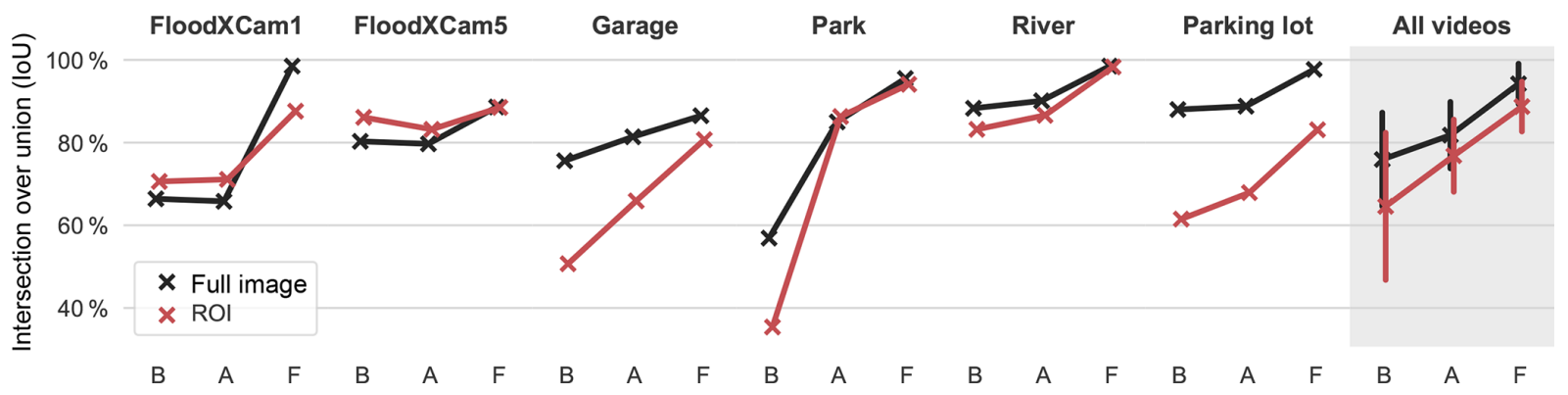

Figure 5. Automatic segmentation performance measured in terms of the intersection over union (IoU) for each video using the Basic (B), Augmented (A), and Fine-tuned (F) DCNNs. On the right side, the overall performance for all videos is depicted, with the vertical segments representing the standard deviation of values.

Compared to the Basic DCNN, the Augmented DCNN provides a visible improvement in most cases. The case of FloodXCam1 is an exception as the DCNN successfully segmented the shallow water flowing on the ground (which was not classified as flooding in the human labels), but did not detect the water ponding in the upper right of the image. This error is fixed in the Fine-tuned DCNN for this video thanks to the use of additional training images from the FloodXCam1 video.

Figure 5 shows the segmentation performance of the DCNNs measured by the IoU, both for the full image and within the defined ROI. The Augmented DCNN improves performance for all videos except for the two FloodX videos. In the case of the Park video, the improvement of IoU for the full image is around 30 percentage points. For the two FloodX videos, however, segmentation seems to suffer slightly under the Augmented network, possibly because the augmentation transformations increased dissimilarity of the training images instead of vice versa. For these two videos, improvement is only achieved thanks to fine-tuning, which proved beneficial for all videos by providing IoUs higher than $90 \%$ on average.

Figure 5 also shows that within the "expert-defined" ROIs, segmentation performance is generally worse. We conclude that a human is generally not able to identify and exclude "difficult" regions of the image a priori, which was one of the original reasons for defining a ROI.

\subsection{Flood level trend extraction}

After the frames of a video are segmented, the SOFI is computed for each frame, providing information about the temporal evolution of the visible flood extent. In Fig. 6, the case of video FloodXCam5 is a clear example of how the SOFI reflects changes in the actual water level. Comparing the SOFI signals to the measured water level, it is evident that a correlation exists, although the relationship does not appear linear. For all DCNN training strategies, the trend of the SOFI signal from the ROI (in red) is easier to visually identify than the trend of the SOFI computed from the whole image (in black), which is advantageous if the signal is to be visually interpreted. However, the SOFI signal from the ROI is also noisier, and does not capture the first flood event occurring at 12:48 local time.

In Fig. 6, we also see that a systematic segmentation error is committed in that water is always falsely detected on the ground between the two events. This example illustrates why our approach focuses on the trend of the SOFI signal and not its absolute values, which are more sensitive to systematic errors.

In Fig. 7, the relationship between the SOFI and the water level is further explored for both FloodX videos. This figure shows how the relationship between SOFI and the water level can be nonlinear, which is a consequence of the topography in which the flooding occurred. For example, in FloodXCam5 a large area of the image is rapidly segmented as water starts covering the floor of the basement, causing an almost vertical segment on the left side of the scatter plot (especially visible for the Fine-tuned network). For the SOFI computed from the full images, we also see systematic and time-variant errors, resulting in portions of the data having a larger internal correlation that are visible as strands of points in Fig. 7.

Generally, it appears that the use of a ROI (red) leads to a stronger association between water level and SOFI. However, the SOFI signals from the ROIs also contain more noise than the SOFI signal derived from the full image. Additionally, in FloodXCam5, it seems that the ROI was poorly selected, resulting in lower sensitivity of SOFI to the water level up to a depth of around $100 \mathrm{~mm}$.

Figure 8 shows the relationship between SOFI and the visually estimated flood intensity in the remaining videos, for which no in situ water level measurement is available. In this figure, the value of the Augmented and Fine-tuned networks appears in the progressive reduction of noise in the SOFI signal.

Two exceptional cases in Fig. 8 need to be explained in more detail. First, the SOFI signal for the River footage suddenly appears to be arbitrary at the highest flood intensity. This is due to intermittent submersion of the camera by 

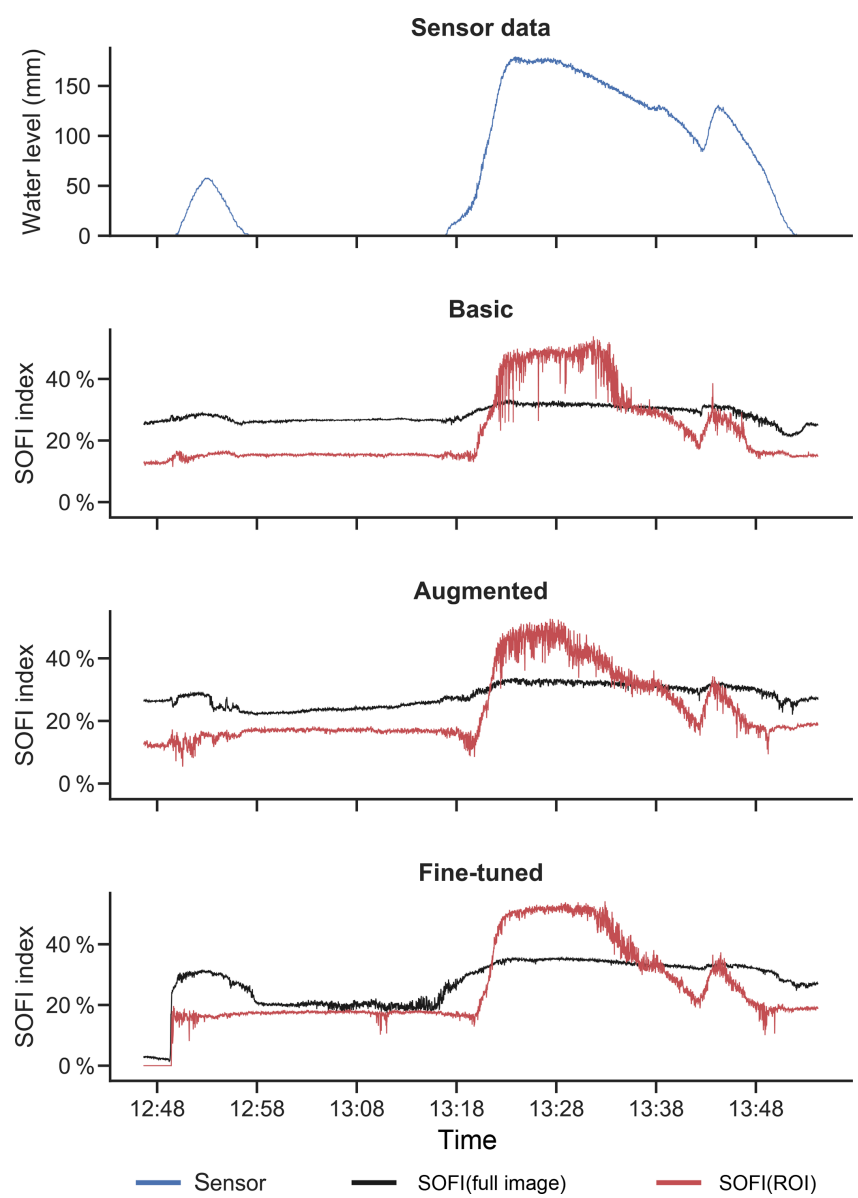

Figure 6. Water level (blue) and SOFI signal for the whole image (black) or the region of interest (ROI, red) for the FloodXCam5 video. With more advanced DCNN training strategies, noise and outliers in SOFI are visibly reduced in the ROI. The time series for the remaining videos can be found in the Supplement (Figs. S2-S6). All time references on the $x$ axis refer to local time.

the floodwater that leads to gross segmentation errors. The Garage video is also exceptional; flooding caused objects to float around in the garage causing constant changes in the visible inundated area and, thus, noise in the SOFI signal.

Figure 9 shows the Spearman correlation coefficients between the SOFI and the flooding intensity for each video and each training strategy. This figure shows that using the Augmented training strategy, the Spearman correlation coefficient for the full image reaches $75 \%$ on average, while for the Fine-tuned training strategy the average correlation coefficient reaches around $85 \%$.

We draw two general conclusions from the results shown in Fig. 9. First, defining a ROI does not consistently improve the ability of the SOFI signal to reproduce flood trends. This could be due to the poorer segmentation performance within the ROIs (Fig. 5), which introduces noise in the water levelSOFI relationship (Figs. 7 and 8). Second, the Fine-tuned networks generally improve the correlation of SOFI with the
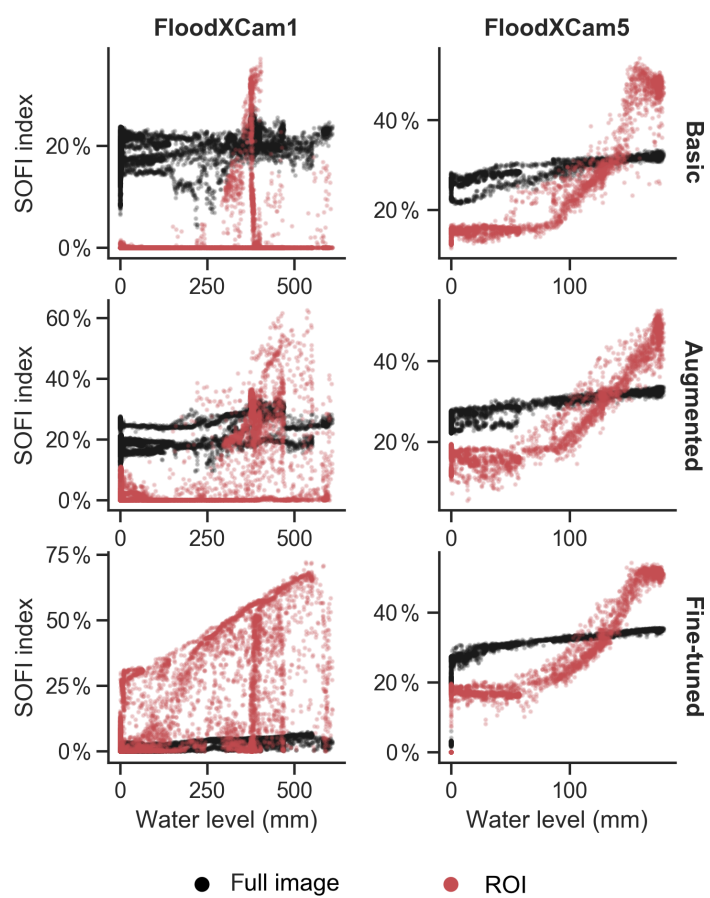

Figure 7. Videos with reference measurements: SOFI computed from full images (black) or from a ROI (red) plotted against measured water level for different training strategies. The reduction of noise and systematic errors with the Fine-tuned strategy is evident.

water level trend, with the exception of the River video. As made visible in Fig. 8, the submersion of the camera leads to frequent outliers that become more distinct from the rest of the signal after fine-tuning, leading to a lower correlation with the water level trend.

\section{Discussion}

\subsection{SOFI as a scalable and robust approach for qualitative water level sensing}

Compared with alternative methods for water level monitoring, our approach is less ambitious in the type of information it aims to provide, as it only attempts to communicate water level fluctuation and not an absolute water level. This weakness is at least partially compensated for by an increased scope of applicability, as it is almost the only surveillance image-based monitoring approach designed to provide water level information without needing to be calibrated to each camera. As described in Sect. 1.3, research has typically been focused on extracting absolute water levels from images, mainly by identifying the water edge in relation to an object that has been measured and manually identified in the image (Bhola et al., 2018; Kim et al., 2011; Sakaino, 2016). Although Lo et al (2015) present a method that does not necessarily need reference measurements, human intervention is still needed for each camera to define virtual markers and a 

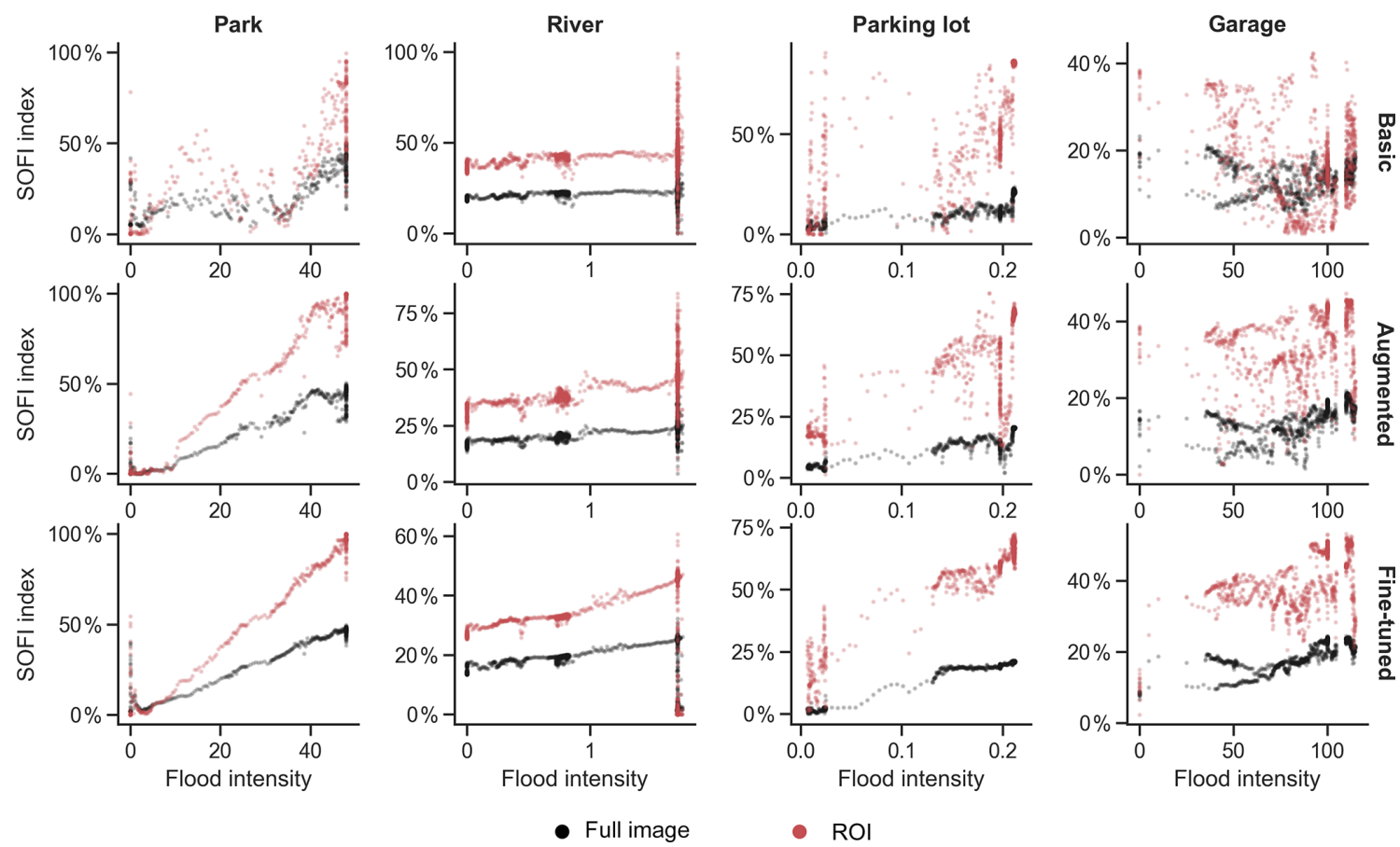

Figure 8. Videos without reference measurements: SOFI computed from full images (black) or from a ROI (red) plotted against visually estimated flood intensity, for different training strategies. There is a visible reduction of noise and increased correlation with the Augmented and Fine-tuned strategies.

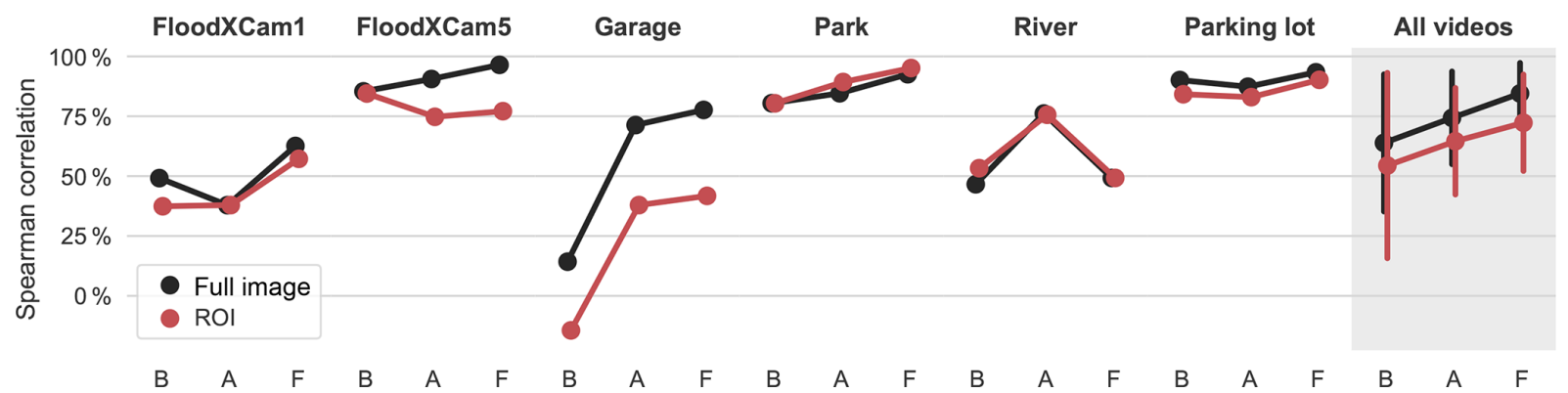

Figure 9. Spearman rank correlation coefficients for each video using the Basic (B), Augmented (A), and Fine-tuned (F) DCNNs. The final category All videos excludes the River video which is an outlier because the camera is intermittently submerged. The vertical segments in the All videos category represent the standard deviation of values. The results show that both the Augmented and Fine-tuned networks improve the correlation of SOFI with the water level, although performance is still varied.

"seed" to inform water segmentation. The concept presented by Jiang et al. (2019) is an exception, theoretically overcoming the need for per-image calibration by taking ubiquitous objects as scale references. Nonetheless, that method assumes that such reference objects are visible in the flooding scene, which is an assumption that may not always hold. In comparison, SOFI does not need specific objects to be present, nor does it need field measurements or manual setup of the image. The DCNN-based water segmentation approach used is flexible enough that given sufficient training data, it can theoretically be applied to any situation. In this study, the scalability of SOFI was demonstrated by applying the Augmented DCNN to six videos not seen during train- ing, from which information was extracted despite complex environments, moving objects, and bad lighting conditions.

The simplicity of the SOFI approach also gives it robustness. By using areal integration to quantify flood intensity, the index is less sensitive to small segmentation errors, which could be problematic if punctual virtual markers are used as in the case of Lo et al. (2015). Also, as SOFI only claims to provide trend information, systematic segmentation errors (e.g., misclassification at the water-background boundary) should only be of minor consequence. This aspect of SOFI is well illustrated in this study by the Park video, for which the SOFI signal had a high correlation with the water level trend despite mediocre segmentation performance. 
Besides the advantages of SOFI, its use of a DCNN carries a disadvantage due to its need for training data. Methods that use heuristics to segment water, such as Lo et al. (2015), do not require as much training data, although their application is then often limited to visually similar situations.

\subsection{Factors influencing the information quality of SOFI}

Although SOFI is theoretically applicable at locations where the extent of visible water correlates with flooding depth, there are certain conditions that can negatively influence the quality of information obtained.

First, floodwater can look very different from case to case. For example, (i) water surface movement can cause a range of different wave structures, (ii) the color of the water is variable and in very agitated flows, bubbles can make the water appear white, (iii) water reflects light that falls on it, at times in a mirror-like fashion, and (iv) surveillance cameras tend to have low color fidelity, dynamic range, resolution, and sharpness. Due to the complexity of segmenting floodwater, one can expect that more training images would be required than for a typical segmentation problem. In our results, the high inter-video variability in segmentation performance suggests that the number of training images should be increased in future studies. In particular, the variability suggests that the training images were not fully representative of the testing images. Thus, it is probable that the segmentation performance could be substantially improved if a larger and more diverse training set were available.

Second, in situations where large quantities of manually labeled images are required, it is inevitable that some labeling errors will occur. Looking into the implications of such errors, Heller et al. (2018) showed that U-net is relatively insensitive to jagged label boundaries. Regarding nonboundary-localized errors, all DCNN architectures investigated were found to be very robust.

Finally, the basic assumption that the extent of visible water correlates with flooding depth may not fully hold for every camera scene. For example, small floods may not be visible due to obstructions, and if the camera is oriented such that the whole image can be covered by water, the highest stages of flooding may be censored from the signal as well. These situations will require special handling when the data are assimilated for model calibration. The scene topography and camera placement also affect the slope and linearity of the SOFI-water depth relationship, and can make the trend more difficult to determine if the correlation in the relationship is small. Additionally, the entry and exit of objects in a flooding scene can compromise the approach, especially if the objects are large and occur with low frequency. The flooding itself may increase the occurrence of such obstructions, for example in the case of cars stuck in traffic or objects transported by the water.

\subsection{Degradation of training image quality improves segmentation of surveillance images}

In this study, the training images available were of higher quality than typical images originating from surveillance cameras, a discrepancy that was expected to limit the performance of the DCNN on surveillance images. Therefore, in the Augmented training strategy, the augmentation step included transformations that lowered the quality of the training images, making them more similar to surveillance images.

The results from the six videos used in this study confirm that the artificial degradation of training image quality not only improves segmentation performance in surveillance footage but also increases the correlation of SOFI with the actual water level trend. While in most videos the improvement was clear, no improvement was observed for the two FloodX videos. These two videos stand out in terms of low image quality, location of water in the upper part of the image, and a different setting surrounded by concrete walls. Therefore, we see a need to investigate such failure cases in order to improve the training data collection and augmentation steps.

\subsection{Fine-tuning of the DCNN to specific cameras}

In situations where segmentation performance is critical, one can fine-tune a general DCNN to a specific surveillance camera. Even with very few additional training images, we find that the segmentation performance and correlation of SOFI with water level trends both improve thanks to fine-tuning. Despite this result, it should be kept in mind that the finetuned DCNNs also lose some generality and may, for example, have issues when lighting conditions are different from those present in the images for fine-tuning.

Our recommendation for fine-tuning is that care should be taken in creating a set of training images that is roughly as diverse as situations in which the fine-tuned DCNN will be used. Additionally, although we performed fine-tuning with very small sets of seven images per video, fine-tuning performance could be further increased by using more images.

\subsection{Regions of interest (ROI) do not deliver the expected value}

The definition of ROIs for SOFI computation, motivated by the possibility of omitting difficult portions of the images and focusing on more information-rich portions, proved unsuccessful. Not only was it difficult for the human "expert" to foresee what area of the image met the above criteria, but a systematic increase of noise in the SOFI signal was observed within the ROI. Therefore, we do not recommend that ROIs be defined systematically for all cameras, but only in cases where multiple hydraulic processes are visible in the image and need to be distinguished. 


\subsection{Practical value of SOFI}

As the qualitative nature and noisiness of SOFI might raise questions about its practical value, two aspects must be recalled. First, SOFI aims to provide information in the context of urban pluvial flood events, for which monitoring data are admittedly difficult to obtain. The studies cited in the introduction have proven that in situations of data scarcity, even qualitative information can be useful in improving model accuracy. In particular, the sparse stream level class information used by van Meerveld et al. (2017) is conceptually equivalent to the flood level trend information contained in SOFI. Second, thanks to the scalability of SOFI, one should be able to apply it to large surveillance networks or retroactively to archived footage at a reasonable cost. By providing data for model calibration and validation, the approach we propose can help reduce modeling uncertainty for urban flood risk assessment and assist in the planning of flood mitigation measures. In addition, the flood level trend information provided by SOFI could help direct the focus of decision-makers and rescue personnel during flood events so that resource use can be optimized. For insurance companies, the flooding information can also help verify claims and establish fair insurance policy premiums.

\subsection{Recommendations for future research}

Future research should assess the actual value of the information provided by SOFI for the validation and calibration of urban flood models. For this, it would be of value to have a large-scale, long-term case study of a flood-prone urban area, in which surveillance footage and reference flood measurements are available. Additionally, possible methods to de-noise the SOFI signal and quantify its reliability should be investigated. In particular, the issue of rapid changes in the SOFI signal due to moving obstructions could be addressed by filtering changes that surpass a given threshold. Another approach to this problem is through automatic definition of ROIs that censor out noise-generating areas of the video scene.

\section{Conclusions}

In this study, we explored the potential of using a deep convolutional neural network (DCNN) and a simple but novel index (namely, SOFI) to obtain flood level trend information from generic surveillance cameras. The results of our study strongly suggest that qualitative flood level information can indeed be extracted automatically and universally from any static camera, although we see the need for many training images to cover the range of appearances that floodwater can take. To compensate for the limited number of training images available in this study, we found that degrading image quality during training improved segmentation performance by approximately $10 \%$ (IoU) on low-quality surveillance im- ages. Additionally, fine-tuning the DCNN to a specific video with as few as seven manually labeled images further improved performance. In our results, the SOFI signal from the camera-independent DCNN correlated with water level trends at a rate of $75 \%$ on average (Spearman rank correlation coefficient). The proof of concept presented in this study has significant implications. Namely, it confirms that footage from surveillance cameras, processed with artificial intelligence, could provide urban flood monitoring data for entire cities while preserving privacy. Without the need for specialized instrumentation or field measurements, monitoring could be conducted at a relatively low cost, which is especially attractive for cities with limited financial resources. Previous research suggests that the qualitative information contained in SOFI can easily be used to reduce parameter uncertainty in urban flood models. Apart from modelingrelated applications, SOFI could also be useful for coordinating flood response and verifying insurance claims.

Code availability. The code used in this work for creating, training, and evaluating the DCNN, as well as extracting the SOFI and plotting results can be found in the following archive: https://doi.org/10.25678/000150 (Moy de Vitry, 2019).

Data availability. The licenses to the images and video data used in this work are held by third parties and cannot be republished by the authors. We encourage interested readers to refer to the references provided for the individual data sources. The DCNN weights trained for each training strategy are available in the following archive: https://doi.org/10.25678/000150 (Moy de Vitry, 2019).

Video supplement. A video demonstration of water detection and SOFI computation can be found at https://doi.org/10.5446/43637 (Moy de Vitry and Kramer, 2019).

Supplement. The supplement related to this article is available online at: https://doi.org/10.5194/hess-23-4621-2019-supplement.

Author contributions. MMdV conceived the method. SK and MMdV implemented the method, analyzed the data, and made the figures. JDW contributed guidance for the DCNN methods. JPL was the principle investigator of the study. MMdV wrote the paper, and all co-authors read the paper and helped with editing.

Competing interests. The authors declare that they have no conflict of interest.

Disclaimer. The funding sponsors had no role in the design of the study; in the collection, analyses, or interpretation of data; in the writing of the manuscript, or in the decision to publish the results. 
Acknowledgements. The authors thank Priyanka Chaudhary for providing the images and annotations used to train the DCNN, as well as Nadine Rüegg for DCNN implementation tips.

Financial support. This research has been supported by the Swiss National Science Foundation (grant no. 169630).

Review statement. This paper was edited by Thomas Kjeldsen and reviewed by Josh Myrans and two anonymous referees.

\section{References}

Abadi, M., Barham, P., Chen, J., Chen, Z., Davis, A., Dean, J., Devin, M., Ghemawat, S., Irving, G., Isard, M., Kudlur, M., Levenberg, J., Monga, R., Moore, S., Murray, D. G., Steiner, B., Tucker, P., Vasudevan, V., Warden, P., Wicke, M., Yu, Y., Zheng, X., Brain, G., Osdi, I., Barham, P., Chen, J., Chen, Z., Davis, A., Dean, J., Devin, M., Ghemawat, S., Irving, G., Isard, M., Kudlur, M., Levenberg, J., Monga, R., Moore, S., Murray, D. G., Steiner, B., Tucker, P., Vasudevan, V., Warden, P., Wicke, M., Yu, Y., and Zheng, X.: TensorFlow: A System for Large-Scale Machine Learning, Proc 12th USENIX Conf. Oper. Syst. Des. Implement., 2-4 November 2016, Savannah, GA, USA, 272-283, 2016.

Bhola, P. K., Nair, B. B., Leandro, J., Rao, S. N., and Disse, M.: Flood inundation forecasts using validation data generated with the assistance of computer vision, J. Hydroinform., 21, 240-256, https://doi.org/10.2166/hydro.2018.044, 2018.

Blanchard, E.: Hurricane Harvey Flooding in Houston, available at: https://www.youtube.com/watch?v=_IBuu06URY (last access: 8 October 2018), 2017.

Chaudhary, P.: Floodwater-estimation through semantic image interpretation, Technical University Munich, Munich, Germany, 2018.

Chaudhary, P., Aronco, S. D., Moy de Vitry, M., Leitao, J. P., and Wegner, J. D.: Flood-Water Level Estimation from Social Media Images, ISPRS Ann. Photogramm. Remote Sens. Spatial Inf. Sci., IV-2/W5, 5-12, 2019.

Chollet, F., and others: Keras Documentation, available at: https: //keras.io (last access: 11 November 2019), 2015.

Cityofchaska: Athletic Park Flood, available at: https://www. youtube.com/watch? $\mathrm{v}=7 \mathrm{cnXTgpenSo}$ (last access: 8 October 2018), 2010.

Cordts, M., Omran, M., Ramos, S., Rehfeld, T., Enzweiler, M., Benenson, R., Franke, U., Roth, S., and Schiele, B.: The Cityscapes Dataset, in: CVPR Workshop on The Future of Datasets in Vision, 7-12 June 2015, Boston, USA, 2015.

El Kadi Abderrezzak, K., Paquier, A., and Mignot, E.: Modelling flash flood propagation in urban areas using a two-dimensional numerical model, Nat. Hazards, 50, 433-460, 2009.

Fukushima, K.: Neocognitron: A self-organizing neural network model for a mechanism of pattern recognition unaffected by shift in position, Biol. Cybern., 36, 193-202, https://doi.org/10.1007/BF00344251, 1980.

Gaitan, S., van de Giesen, N. C., and ten Veldhuis, J. A. E. E.: Can urban pluvial flooding be predicted by open spatial data and weather data?, Environ. Modell. Softw., 85, 156-171, https://doi.org/10.1016/j.envsoft.2016.08.007, 2016.

Goold, B., Van Den Hoven, J., Lim, L., Seck, M., Squires, P., and Töpfer, E.: Citizens, Cities and Video Surveillance, European Forum for Urban Security, Paris, France, 2010.

He, K., Zhang, X., Ren, S., and Sun, J.: Deep Residual Learning for Image Recognition, in: 2016 IEEE Conference on Computer Vision and Pattern Recognition (CVPR), 26 June-1 July 2016, Las Vegas, USA, 770-778, 2016.

He, K., Gkioxari, G., Dollar, P., Girshick, R., Dollár, P., and Girshick, R.: Mask R-CNN, in: Proceedings of the IEEE International Conference on Computer Vision, 22-29 October 2017, Venice, Italy, 2017.

Heller, N., Dean, J., and Papanikolopoulos, N.: Imperfect Segmentation Labels: How Much Do They Matter?, in: Intravascular Imaging and Computer Assisted Stenting and Large-Scale Annotation of Biomedical Data and Expert Label Synthesis, Springer International Publishing, 112-120, available at: https:// link.springer.com/chapter/10.1007/978-3-030-01364-6_13 (last access: 14 November 2019), 2018.

Hunter, N. M., Bates, P. D., Neelz, S., Pender, G., Villanueva, I., Wright, N. G., Liang, D., Falconer, R. A., Lin, B., Waller, S., Crossley, A. J., and Mason, D. C.: Benchmarking 2D hydraulic models for urban flooding, Proc. Inst. Civ. Eng.-Water Manag., 161, 13-30, https://doi.org/10.1680/wama.2008.161.1.13, 2008.

Hurricanetrack: Brays Bayou Flooding Time Lapse and RealTime Archive, available at: https://www.youtube.com/watch?v= y6jByqVX7PE (last access: 8 October 2018), 2015.

Ioffe, S. and Szegedy, C.: Batch Normalization: Accelerating Deep Network Training by Reducing Internal Covariate Shift, arXiv1502.03167, available at: http://arxiv.org/abs/1502.03167 (last access: 13 November 2019), 2015.

Jiang, J., Liu, J., Qin, C.-Z. Z., and Wang, D.: Extraction of Urban Waterlogging Depth from Video Images Using Transfer Learning, Water, 10, 1485, https://doi.org/10.3390/w10101485, 2018.

Jiang, J., Liu, J., Cheng, C., Huang, J., and Xue, A.: Automatic Estimation of Urban Waterlogging Depths from Video Images Based on Ubiquitous Reference Objects, Remote Sens., 11, 587, https://doi.org/10.3390/rs11050587, 2019.

Jiang, Y., Zevenbergen, C., and Ma, Y.: Urban pluvial flooding and stormwater management: A contemporary review of China's challenges and "sponge cities" strategy, Environ. Sci. Policy, 80, 132-143, https://doi.org/10.1016/j.envsci.2017.11.016, 2018.

Kim, B., Sanders, B. F., Han, K., Kim, Y., and Famiglietti, J. S.: Calibration of Stormwater management model using flood extent data, Proc. Inst. Civ. Eng. Water Manag., 167, 17-29, https://doi.org/10.1680/wama.12.00051, 2014.

Kim, J., Han, Y., and Hahn, H.: Embedded implementation of image-based water-level measurement system, IET Comput. Vis., 5, 125-133, https://doi.org/10.1049/Iet-Cvi.2009.0144, 2011.

Kingma, D. P. and Ba, J. L.: Adam: A method for stochastic gradient descent, ICLR Int. Conf. Learn. Represent., 7-9 May 2015, San Diego, CA, USA, 1-15, 2015.

Leandro, J., Chen, A. S., Djordjević, S., and Savić, D. A.: Comparison of 1D/1D and 1D/2D Coupled (Sewer/Surface) Hydraulic Models for Urban Flood Simulation, J. Hydraul. Eng., 135, 495-504, https://doi.org/10.1061/(ASCE)HY.19437900.0000037, 2009. 
Levandowsky, M. and Winter, D.: Distance between Sets, Nature, 234, 34-35, https://doi.org/10.1038/234034a0, 1971.

Liu, L., Liu, Y., Wang, X., Yu, D., Liu, K., Huang, H., and $\mathrm{Hu}, \mathrm{G} .:$ Developing an effective 2-D urban flood inundation model for city emergency management based on cellular automata, Nat. Hazards Earth Syst. Sci., 15, 381-391, https://doi.org/10.5194/nhess-15-381-2015, 2015.

Lo, S. W., Wu, J. H., Lin, F. P., and Hsu, C. H.: Visual sensing for urban flood monitoring, Sensors (Switzerland), 15, 2000620029, https://doi.org/10.3390/s150820006, 2015.

Long, J., Shelhamer, E., and Darrell, T.: Fully Convolutional Networks for Semantic Segmentation, in: Proceedings of the IEEE Conference on Computer Vision and Pattern Recognition, 712 June 2015, Boston, MA, USA, 3431-3440, 2015.

Lv, Y., Gao, W., Yang, C., and Wang, N.: Inundated Areas Extraction Based on Raindrop Photometric Model (RPM) in Surveillance Video, Water, 10, 1332, https://doi.org/10.3390/w10101332, 2018.

McKinney, W.: Data Structures for Statistical Computing in Python, in: Proceedings of the 9th Python in Science Conference, 28 June-3 July 2010, Austin, Texas, USA, 2010.

Mousa, M., Zhang, X., and Claudel, C.: Flash Flood Detection in Urban Cities Using Ultrasonic and Infrared Sensors, IEEE Sens. J., 16, 7204-7216, https://doi.org/10.1109/JSEN.2016.2592359, 2016.

Moy de Vitry, M.: Data for: Scalable Flood Level Trend Monitoring with Surveillance Cameras using a Deep Convolutional Neural Network, Zenodo, https://doi.org/10.25678/000150, 2019.

Moy de Vitry, M. and Kramer, S.: Water level trend monitoring with a surveillance camera using a deep convolutional neural network, https://doi.org/10.5446/43637, 2019.

Moy de Vitry, M., Dicht, S., and Leitão, J. P.: floodX: urban flash flood experiments monitored with conventional and alternative sensors, Earth Syst. Sci. Data, 9, 657-666, https://doi.org/10.5194/essd-9-657-2017, 2017.

Noh, H., Hong, S., and Han, B.: Learning Deconvolution Network for Semantic Segmentation, in: 2015 IEEE International Conference on Computer Vision (ICCV), 2015 Inter, Santiago, Chile, 7-13 December 2015, 1520-1528, 2015.

Paprotny, D., Sebastian, A., Morales-Nápoles, O., and Jonkman, S. N.: Trends in flood losses in Europe over the past 150 years, Nat. Commun., 9, 1985, https://doi.org/10.1038/s41467-018-042531, 2018.

Pröve, P.-L.: Unet-keras, available at: https://github.com/pietz/ unet-keras (last access: 13 November 2019), 2017.

Roisman, D.: Houston Harvey Flood - Meyerland Neighborhood - August 272017 - Garage Time Lapse, available at: https:// www.youtube.com/watch?v=ZOpWO7rJbtU (last access: 8 October 2018), 2017.

Ronneberger, O., Fischer, P., and Brox, T.: U-net: Convolutional networks for biomedical image segmentation, Lect. Notes Comput. Sci. (including Subser. Lect. Notes Artif. Intell. Lect. Notes Bioinformatics), 9351, 234-241, https://doi.org/10.1007/978-3319-24574-4_28, 2015.

Sakaino, H.: Camera-Vision-Based Water Level Estimation, IEEE Sens. J., 16, 7564-7565, https://doi.org/10.1109/JSEN.2016.2603524, 2016.
Seibert, J.: Multi-criteria calibration of a conceptual runoff model using a genetic algorithm, Hydrol. Earth Syst. Sci., 4, 215-224, https://doi.org/10.5194/hess-4-215-2000, 2000.

Skougaard Kaspersen, P., Høegh Ravn, N., Arnbjerg-Nielsen, K., Madsen, H., and Drews, M.: Comparison of the impacts of urban development and climate change on exposing European cities to pluvial flooding, Hydrol. Earth Syst. Sci., 21, 4131-4147, https://doi.org/10.5194/hess-21-4131-2017, 2017.

Spearman, C.: The Proof and Measurement of Association between Two Things, Am. J. Psychol., 15, 72-101, https://doi.org/10.2307/1412159, 1904.

Sperber, S., Seck, M., and Johnston, E.: Surveille Deliverable 2.3: Paper by local authorities end-users, available at: https://surveille.eui.eu/wp-content/uploads/sites/19/2015/04/ D2.3-Paper-by-Local-Authorities-End-Users.pdf (last access: 13 November 2019), 2013.

ten Veldhuis, J. A. E.: How the choice of flood damage metrics influences urban flood risk assessment, J. Flood Risk Manag., 4, 281287, https://doi.org/10.1111/j.1753-318X.2011.01112.x, 2011.

Tscheikner-Gratl, F., Zeisl, P., Kinzel, C., Leimgruber, J., Ertl, T., Rauch, W., and Kleidorfer, M.: Lost in calibration: why people still don't calibrate their models, and why they still should - a case study from urban drainage modelling, Water Sci. Technol., 395, 2337-2348, https://doi.org/10.2166/wst.2016.395, 2016.

van Meerveld, H. J. I., Vis, M. J. P., and Seibert, J.: Information content of stream level class data for hydrological model calibration, Hydrol. Earth Syst. Sci., 21, 4895-4905, https://doi.org/10.5194/hess-21-4895-2017, 2017.

van Riel, W.: Exploratory study of pluvial flood impacts in Dutch urban areas, Deltares, Delft, the Netherlands, 2011.

Wang, R. Q., Mao, H., Wang, Y., Rae, C., and Shaw, W.: Hyper-resolution monitoring of urban flooding with social media and crowdsourcing data, Comput. Geosci., 111, 139-147, https://doi.org/10.1016/j.cageo.2017.11.008, 2018.

Wani, O., Scheidegger, A., Carbajal, J. P., Rieckermann, J., and Blumensaat, F.: Parameter estimation of hydrologic models using a likelihood function for censored and binary observations, Water Res., 121, 290-301, https://doi.org/10.1016/j.watres.2017.05.038, 2017.

Yu, D., Yin, J., and Liu, M.: Validating city-scale surface water flood modelling using crowd-sourced data, Environ. Res. Lett., 11, 124011, https://doi.org/10.1088/1748-9326/11/12/124011, 2016.

Zahnt, N., Eder, M., and Habersack, H.: Herausforderungen durch pluviale Überflutungen - Grundlagen, Schäden und Lösungsansätze, Osterr. Wasser Abfallwirtsch., 70, 64-77, 2018.

Zeiler, M. D., Taylor, G. W., and Fergus, R.: Adaptive deconvolutional networks for mid and high level feature learning, in: Proceedings of the IEEE International Conference on Computer Vision, 6-13 November 2011, Barcelona, Spain, 2018-2025, 2011.

Zou, K. H., Warfield, S. K., Bharatha, A., Tempany, C. M. C., Kaus, M. R., Haker, S. J., Wells, W. M., Jolesz, F. A., and Kikinis, R.: Statistical Validation of Image Segmentation Quality Based on a Spatial Overlap Index, Acad. Radiol., 11, 178-189, https://doi.org/10.1016/S1076-6332(03)00671-8, 2004. 